\title{
Ignoranz oder Irrelevanz? - Direkte Demokratie auf österreichisch
}

\author{
Harald Eberhard/Konrad Lachmayer
}

\section{Einleitung}

Art. 1 der österreichischen Verfassung (B-VG) ${ }^{1}$ besagt: „Österreich ist eine demokratische Republik. Ihr Recht geht vom Volk aus". Diese Bestimmung wird in der herrschenden Rechtsprechung ${ }^{2}$ und Lehre ${ }^{3}$ als bloße Proklamation verstanden. Die konkreten Inhalte des demokratischen Prinzips, das als eines der „Grundprinzipien“ der österreichischen Verfassung gilt und dessen umfassendere Modifikationen daher eine volksabstimmungspflichtige „Gesamtänderung“ der Verfassung 4 i.S.d. Art. 44 Abs. 3 B-VG $\mathrm{VG}^{5}$ darstellen würden, ergeben sich aus den weiteren Bestimmungen der Verfassung, etwa jenen über Wahlen ${ }^{6}$ und die Ausgestaltung der Gesetzgebung. ${ }^{7}$ Dieses Gesamtbild zeigt ein System mit einem dominierenden Zug repräsentativer Demokratie, einer starken Stellung der Parlamente als allgemeine Vertretungskörper sowohl in der Gesetzgebung als auch - über die Weisungsbindung der Verwaltung

1 Bundes-Verfassungsgesetz (B-VG), BGB1. I 1930/1. Diese „Kern“-Verfassungsurkunde wurde seither über hundertmal novelliert. Mangels eines Inkorporationsgebotes erlaubt die österreichische Verfassung auch die Schaffung fugitiver Verfassungsbestimmungen in einfachen Bundes- und Landesgesetzen, früher - vor der mit 1.1.2008 in Kraft getretenen Novelle BGB1. I 2008/2 - auch in Staatsverträgen (vgl. dazu Art. 44 Abs. 1 B-VG: „Verfassungsgesetze oder in einfachen Gesetzen enthaltene Verfassungsbestimmungen können vom Nationalrat nur in Anwesenheit von mindestens der Hälfte der Mitglieder und mit einer Mehrheit von zwei Dritteln der abgegebenen Stimmen beschlossen werden; sie sind als solche [,Verfassungsgesetz', ,Verfassungsbestimmung'] ausdrücklich zu bezeichnen.").

2 Vgl. etwa VfSlg. 15.886/2000, 17.264/2004 m.w.N.

3 Vgl. etwa T. Öhlinger, Verfassungsrecht, 8. Aufl. 2009, Rz. 341; H. Mayer, Das österreichische Bundesverfassungsrecht. Kurzkommentar, 4. Aufl. 2007, S. 3, jeweils m.w.N.; a.A. P. Oberndorfer, Art. 1 B-VG, in: K. Korinek/M. Holoubek (Hrsg.), Österreichisches Bundesverfassungsrecht. Kommentar (3. Lfg. 2000), Rz. 8.

4 Der Begriff der „Gesamtänderung der Bundesverfassung“ war seit jeher strittig (dazu eingehend etwa P. Pernthaler, Österreichisches Bundesstaatsrecht, 2004, S. 32 f., 125, 678 f.; Öhlinger, Verfassungsrecht [Fn. 3], Rz. 64 f.). Eine unzweifelhafte Gesamtänderung stellte bislang ausschließlich die Änderung des demokratischen, bundesstaatlichen und rechtsstaatlichen Prinzips durch den im Jahr 1995 erfolgten Beitritt Österreichs zur Europäischen Union dar. Über das BVG, mit dem zum Abschluss des Beitrittsvertrages ermächtigt wurde (BGB1. 1994/744), ist daher am 12.6.1994 eine Volksabstimmung abgehalten worden.

5 „Jede Gesamtänderung der Bundesverfassung, eine Teiländerung aber nur, wenn dies von einem Drittel der Mitglieder des Nationalrates oder des Bundesrates verlangt wird, ist nach Beendigung des Verfahrens gemäß Art. 42, jedoch vor der Beurkundung durch den Bundespräsidenten, einer Abstimmung des gesamten Bundesvolkes zu unterziehen.“

6 Vgl. Art. 26, 95 und 117 B-VG betreffend die Wahlgrundsätze für die Wahlen zum Nationalrat, zu den Landtagen (den Länderparlamenten) und den Gemeinderäten (den allgemeinen Vertretungskörpern auf der dritten Ebene des Bundesstaates, den Gemeinden).

7 Vgl. Art. 41 B-VG (,Weg der Bundesgesetzgebung“). 
gegenüber den obersten Organen der Vollziehung ${ }^{8}$ - bei der Vollziehung. Letzteres zeigt sich in der politischen und rechtlichen Verantwortlichkeit der obersten Organe der Verwaltung gegenüber diesen Parlamenten. ${ }^{9}$ Dieses (Selbst-)Verständnis spiegelt eine Facette österreichischer Demokratie wider, die durch ihre Grundentscheidung für ein repräsentatives Modell von Demokratie direkt-demokratische Instrumente traditionell an den Rand gedrängt hat. Art. 1 B-VG stellt damit zwar deutlich klar, dass das Recht vom Volk ausgeht und somit die demokratische Legitimation aus dem Volk zu ziehen ist, die Ausgestaltung der österreichischen Demokratie erfolgt in den Art. 1 B-VG folgenden Verfassungsbestimmungen aber durch ein System der repräsentativen Demokratie, das die Rolle des Volkes auf eine theoretische Legitimationsbasis reduziert, wobei dessen Bedeutung durch das Wahlrecht institutionalisiert, formalisiert und beschränkt ist. Elemente der direkten Demokratie setzen aber gerade daran an und intendieren, die Mitwirkung des Volkes an der Gesetzgebung auf konkrete Gesetzesbeschlüsse und deren in ihrem Vorfeld notwendige politische Prozesse zu fokussieren und insoweit auch auf die Zeit zwischen den Wahlen zu erstrecken. ${ }^{10}$

Es entspricht der herrschenden Auffassung der Staatsrechtslehre, ${ }^{11}$ dass das B-VG eine Systementscheidung für die repräsentative Demokratie mit untergeordneten plebiszitären Elementen realisiert, d.h. - mit den Worten Pernthalers ${ }^{12}$ - einen „Vorrang der Repräsentation vor den Volksrechten“ oder auch ein System (bloß) „semidirekter Demokratie“. Es wird in der Dogmatik auch das Spannungsverhältnis zum rechtsstaatlichen Prinzip betont, in das Elemente der direkten Demokratie dann treten können, wenn damit Einzelfälle entschieden würden, ${ }^{13}$ die im Rechtsstaat

8 Art. 20 Abs. 1 B-VG: „Unter der Leitung der obersten Organe des Bundes und der Länder führen nach den Bestimmungen der Gesetze auf Zeit gewählte Organe, ernannte berufsmäßige Organe oder vertraglich bestellte Organe die Verwaltung. Sie sind den ihnen vorgesetzten Organen für ihre amtliche Tätigkeit verantwortlich und, soweit in Gesetzen gemäß Abs. 2 nicht anderes bestimmt ist, an deren Weisungen gebunden. Das nachgeordnete Organ kann die Befolgung einer Weisung ablehnen, wenn die Weisung entweder von einem unzuständigen Organ erteilt wurde oder die Befolgung gegen strafgesetzliche Vorschriften verstoßen würde.“ Art. 20 Abs. 2 B-VG sieht seit der B-VG-Novelle BGB1. I 2008/2 allerdings die (zuvor nicht vorhandene) Ermächtigung des einfachen Gesetzgebers vor, bestimmte typisiert normierte Gruppen von Verwaltungsorganen weisungsfrei zu stellen.

9 Vgl. Art. 74 und 76i.V.m. 142 B-VG.

10 Pernthaler, Bundesstaatsrecht (Fn. 4), S. 86: „Zur Repräsentation gehört ... nicht nur der Formalakt der Wahl, sondern auch das dadurch geschaffene Verantwortungs- und Vertrauensverhältnis (demokratischer Konsens) zwischen Repräsentanten und Volk.“

11 Vgl. etwa M. Welan, Art. 1 B-VG, in: FS Adamovich, 1992, S. 721 ff. m.w.N.

12 Pernthaler, Bundesstaatsrecht (Fn. 4), S. 84 f.

13 Nicht anders kann folgende Formulierung in Art. 49 b Abs. 1 B-VG im Hinblick auf die Volksbefragung gedeutet werden: „Wahlen sowie Angelegenheiten, über die ein Gericht oder eine Verwaltungsbehörde zu entscheiden hat, können nicht Gegenstand einer Volksbefragung sein." 
idealtypisch durch eine streng gesetzesgebundene Behörde oder durch ein Gericht entschieden werden müssten. ${ }^{14}$

\section{Direkt-demokratische Instrumente in systematischer Zusammenschau}

\section{a) Überblick}

Die gemachten Ausführungen lassen zunächst annehmen, dass direkte Demokratie in der österreichischen Verfassung nicht oder nur marginal geregelt ist. Dem ist aber nicht so. Es finden sich - seit der Stammfassung des B-VG - die (klassischen) Elemente direkter Demokratie wie die Volksabstimmung ${ }^{15}$ und das Volksbegehren ${ }^{16}$ sowie - seit $1989^{17}$ - die Volksbefragung ${ }^{18}$ sowohl in der österreichischen Verfas-

14 Dazu etwa L.K. Adamovich, Demokratie und Rechtsstaat, in: FS Rosenzweig, 1988, S. 27 (33 f.); speziell für direkt-demokratische Instrumente auf Ebene der Vollziehung H. Mayer, Plebiszitäre Instrumente in der staatlichen Willensbildung, in: FS 75 Jahre Bundesverfassung, 1995, S. 341 (358 f.: ,,in einem Rechtsstaat ist ... für plebiszitäre Elemente in der Vollziehung nur wenig Raum“").

15 Art. 43 B-VG: „Einer Volksabstimmung ist jeder Gesetzesbeschluss des Nationalrates nach Beendigung des Verfahrens gemäß Art. 42, jedoch vor seiner Beurkundung durch den Bundespräsidenten, zu unterziehen, wenn der Nationalrat es beschließt oder die Mehrheit der Mitglieder des Nationalrates es verlangt.“ - Siehe auch Art. 44 Abs. 3 B-VG: „Jede Gesamtänderung der Bundesverfassung, eine Teiländerung aber nur, wenn dies von einem Drittel der Mitglieder des Nationalrates oder des Bundesrates verlangt wird, ist nach Beendigung des Verfahrens gemäß Art. 42, jedoch vor der Beurkundung durch den Bundespräsidenten, einer Abstimmung des gesamten Bundesvolkes zu unterziehen."

16 Art. 41 Abs. 2 B-VG: „Jeder von 100000 Stimmberechtigten oder von je einem Sechstel der Stimmberechtigten dreier Länder gestellte Antrag (Volksbegehren) ist von der Bundeswahlbehörde dem Nationalrat zur Behandlung vorzulegen. Stimmberechtigt bei Volksbegehren ist, wer am letzten Tag des Eintragungszeitraums das Wahlrecht zum Nationalrat besitzt und in einer Gemeinde des Bundesgebietes den Hauptwohnsitz hat. Das Volksbegehren muss eine durch Bundesgesetz zu regelnde Angelegenheit betreffen und kann in Form eines Gesetzesantrages gestellt werden.“

17 Vgl. die mit 1.7.1989 in Kraft getretene B-VG-Novelle BGB1. 1988/685.

18 Art. 49 b B-VG: ,(1) Eine Volksbefragung über eine Angelegenheit von grundsätzlicher und gesamtösterreichischer Bedeutung, zu deren Regelung die Bundesgesetzgebung zuständig ist, hat stattzufinden, sofern der Nationalrat dies auf Grund eines Antrages seiner Mitglieder oder der Bundesregierung nach Vorberatung im Hauptausschuss beschließt. Wahlen sowie Angelegenheiten, über die ein Gericht oder eine Verwaltungsbehörde zu entscheiden hat, können nicht Gegenstand einer Volksbefragung sein.

(2) Ein Antrag gemäß Abs. 1 hat einen Vorschlag für die der Volksbefragung zugrunde zu legende Fragestellung zu enthalten. Diese hat entweder aus einer mit ,ja' oder ,nein` zu beantwortenden Frage oder aus zwei alternativen Lösungsvorschlägen zu bestehen.

(3) Volksbefragungen sind unter sinngemäßer Anwendung von Art. 45 und 46 durchzuführen. Stimmberechtigt bei Volksbefragungen ist, wer am Befragungstag das Wahlrecht zum Nationalrat besitzt. Die Bundeswahlbehörde hat das Ergebnis einer Volksbefragung dem Nationalrat sowie der Bundesregierung vorzulegen." 
sung ${ }^{19}$ als auch in einfachgesetzlichen Ausführungsregelungen. ${ }^{20}$ Erst jüngere Entwicklungen wie die Möglichkeit, Bürgermeister auf Gemeindeebene direkt zu wählen, ${ }^{21}$ oder die Ermächtigung des Landesgesetzgebers zur Schaffung direkt-demokratischer Elemente in Angelegenheiten des eigenen Wirkungsbereiches der Gemeinde ${ }^{22}$ belegen, dass es auf verfassungsrechtlicher Ebene sowohl Anknüpfungspunkte als auch eine - zwar beschränkte, aber doch mit Blick auf den Ausbau dieser Elemente vorhandene - Dynamik im Zusammenhang mit direkter Demokratie gibt. Diese Dynamik zeigt sich auch in einer bemerkenswerten Kasuistik der landesverfassungsgesetzlichen Bestimmungen und einfachgesetzlichen Regelungen im Landesrecht, die direkt-demokratische Elemente sowohl auf Ebene der Gesetzgebung als auch - v.a. mit Blick auf die dritte Ebene des Bundesstaates - auf jener der Gemeinden vorsehen. ${ }^{23}$ Die landesverfassungsrechtlichen Regelungen dürfen aber im Lichte der (in dieser Hinsicht umstrittenen ${ }^{24}$ ) Judikatur des Verfassungsgerichtshofes (VfGH $)^{25}$ in ihrer Intensität - d.h. in ihrer Relation zu den repräsentativ-demokratischen Institutionen - nicht über jenes Maß hinausgehen, das das B-VG für die Ebene der Bundesgesetzgebung normiert. Ein Volksbegehren, auch wenn es von einer qualifizierten Mehrheit unterstützt wird, darf etwa nicht zu einer Bindung des jeweiligen Parlaments führen. ${ }^{26}$ Diese stringente Sichtweise nährt sich aus einem bestimmten „Vorverständnis“ der bundesverfassungsgesetzlichen Regelungen ${ }^{27}$ über direkte Demokratie und deren Übertragung auf die Landes- und Gemeindeebene, obwohl es gerade für diese beiden Ebenen keine vergleichbaren - auch als Einschränkung (!) direkter Demokratie zu verstehenden - Regelungen gibt. ${ }^{28}$

19 Zusammenfassend vgl. etwa Öhlinger, Verfassungsrecht (Fn. 3), Rz. 451 ff.; R. Walter/H. Mayer/G. Kucsko-Stadlmayer, Grundriss Bundesverfassungsrecht, 10. Aufl. 2007, Rz. 437/1, 442 ff., 463 ff.; L.K. Adamovich/B.C. Funk/G. Holzinger, Österreichisches Staatsrecht, Band 2: Staatliche Organisation, 1998, Rz. 21.060 ff., 21.078, 21.085.

20 Vgl. dazu das Volksbegehrengesetz 1973 BGBl. 1973/344, Volksabstimmungsgesetz 1972 BGB1. 1973/79 und Volksbefragungsgesetz 1989 BGB1. 1989/356. Für Detailfragen zu den einzelnen Instrumenten und den genannten Ausführungsregelungen vgl. T. Thanner, Das Recht der direkten Demokratie, 2007.

21 Diese Ermächtigung besteht erst seit der B-VG-Novelle BGB1. 1994/504. Vgl. Art. 117 Abs. 6 B-VG: „Der Bürgermeister wird vom Gemeinderat gewählt. In der Landesverfassung kann vorgesehen werden, dass die zur Wahl des Gemeinderates Berechtigten den Bürgermeister wählen. In diesem Fall ist Art. 26 Abs. 6 sinngemäß anzuwenden.“

22 Diese Ermächtigung wurde erst mit der B-VG-Novelle BGB1. 1984/490 geschaffen. Siehe nunmehr Art. 117 Abs. 8 B-VG: ,In Angelegenheiten des eigenen Wirkungsbereiches der Gemeinde kann der Landesgesetzgeber die unmittelbare Teilnahme und Mitwirkung der zum Gemeinderat Wahlberechtigten vorsehen."

23 Vgl. dazu den Überblick bei Öhlinger, Verfassungsrecht (Fn. 3), Rz. 456 f.; Pernthaler, Bundesstaatsrecht (Fn. 4), S. $90 \mathrm{ff}$.

24 Dazu vor allem T. Öhlinger, Bundesverfassungsrechtliche Grenzen der Volksgesetzgebung, in: Montfort, Vierteljahresschrift für Geschichte und Gegenwart Vorarlbergs 2000, S. 402 ff.;

A. Gamper, Demokratie und bundesstaatliches Homogenitätsprinzip, in: ÖJZ 2003, $441 \mathrm{ff}$.

25 Dazu sogleich unter 3.

$26 \mathrm{Zu}$ diesem Problemkreis näher unten $2 \mathrm{c}$ ) und $4 \mathrm{~b}$ ).

$27 \mathrm{Zu}$ diesem Gedanken Pernthaler, Bundesstaatsrecht (Fn. 4), S. 42, 67.

28 I.d.S. treffend Gamper, Demokratie (Fn. 24), S. $441 \mathrm{ff}$. 


\section{b) Volksbefragung}

Jedenfalls für die Ebene des Bundesverfassungsrechts ergibt sich dabei folgendes Bild: die Volksbefragung (als jüngstes der direkt-demokratischen Instrumente) ist ein Element zur Ermittlung des Volkswillens im Vorfeld gesetzgeberischer Initiativen $^{29}$ in einer „Angelegenheit von grundsätzlicher und gesamtösterreichischer Bedeutung, zu deren Regelung die Bundesgesetzgebung zuständig ist" (Art. $49 \mathrm{~b}$ $\mathrm{B}-\mathrm{VG}) .^{30}$ Es ist insoweit eine Entscheidungshilfe für den parlamentarischen Gesetzgeber, ${ }^{31}$ dem das Ergebnis der Volksbefragung - ebenso wie der Bundesregierung - vorzulegen ist. Sowohl die Formulierung der Fragestellung (,agenda setting $\left.^{\text {" }}\right)^{32}$ als auch die Anordnung einer Volksbefragung selbst liegen bei staatlichen Organen (Nationalrat [1. Kammer im österreichischen Parlament, entspricht dem deutschen Bundestag $]^{33}$ und Bundespräsident ${ }^{34}$ ), die Willensäußerung des Volkes hat daher reaktiven Charakter. ${ }^{35}$ Kennzeichnendes Merkmal ist zudem die rechtlich unverbindliche, somit ausschließlich politische Wirkung der Volksbefragung. ${ }^{36}$ Aus faktischer Sicht muss deren Charakter als „totes Verfassungsrecht“ betont werden, zumal es bislang keine derartige bundesweite Volksbefragung gegeben hat.

\section{c) Volksbegehren}

Das (von zumindest 100.000 Stimmberechtigten) unterstützte Volksbegehren ${ }^{37}$ (Art. 41 Abs. 2 B-VG) als (formal gegenüber anderen Formen der Gesetzesinitiative gleichberechtigte) Form der Gesetzesinitiative (entweder in Form eines Gesetzesentwurfes oder auch einer bloßen Anregung) ist dem Nationalrat vorzulegen. Im

29 Dazu Adamovich/Funk/Holzinger, Österreichisches Staatsrecht 2 (Fn. 19), Rz. 21.085.

30 Zur Reichweite dieser Formulierung (im Besonderen im Hinblick auf verfassungsrechtliche Änderungen) und zu verfahrensrechtlichen Fragen vgl. näher $R$. Thienel, Verfassungsfragen der Volksbefragung nach Art. 49 b B-VG - Dargestellt am Beispiel der geplanten EU-Volksbefragung, in: JRP 2000, 327 ff.; H. Mayer, Welche Volksbefragung erlaubt die Verfassung?, in: JRP 2001, 113 ff.; R. Thienel, Nochmals: Verfassungsfragen der Volksbefragung, in: JRP 2001, $117 \mathrm{ff}$.

31 Mayer, Plebiszitäre Elemente (Fn. 14), S. 354.

32 Vgl. Art. 49 b Abs. 2 B-VG: „Der Antrag auf Durchführung einer Volksbefragung hat einen Vorschlag für die der Volksbefragung zugrunde zu legende Fragestellung zu enthalten. Diese hat entweder aus einer mit ,ja' oder ,nein' zu beantwortenden Frage oder aus zwei alternativen Lösungsvorschlägen zu bestehen.“

33 Erforderlich ist ein Beschluss des Nationalrates auf Grund eines Antrages seiner Mitglieder (mindestens 5) oder der Bundesregierung.

34 Art. 49 b Abs. 3 B-VG i.V.m. Art. 46 Abs. 1 B-VG.

35 F. Merli, Art. 41/2 B-VG, in: K. Korinek/M. Holoubek (Hrsg.), Österreichisches Bundesverfassungsrecht. Kommentar (1. Lfg. 1999), Rz. 9.

36 Mayer, Plebiszitäre Elemente (Fn. 14), S. 354.

37 Um ein Volksbegehren überhaupt einleiten zu können, muss der entsprechende Antrag auf Einleitung eines Volksbegehrens von einem Promille der Wohnbevölkerung (derzeit 8.032 Personen) unterstützt sein (vgl. § 3 Abs. 2 Volksbegehrengesetz 1973). 
Unterschied zur Volksbefragung und zur Volksabstimmung hat das Volksbegehren damit - sieht man vom Fall einer offensiven Forcierung durch die mit dem Bundesgesetzgeber identen politischen Parteien $\mathrm{ab}^{38}$ - keinen bloß reaktiven Charakter. Es löst aber unabhängig vom Grad seiner Unterstützung nur die geschäftsordnungsmäßige Behandlung im Nationalrat aus. ${ }^{39}$ Es kommt ihm im Besonderen keinerlei rechtliche Verbindlichkeit zu. ${ }^{40}$ Diskutierte Ansätze ${ }^{41}$ einer rechtlichen Verknüpfung von (unverbindlichen, aber stark unterstützten) Volksbegehren und (verbindlicher) Volksabstimmung verliefen bisher im Sand. ${ }^{42}$

\section{d) Volksabstimmung}

Chronologisch im spätesten Teil des Gesetzgebungsverfahrens angesiedelt ist die Volksabstimmung, die nach Beendigung des Verfahrens im Nationalrat und Bundesrat, aber vor der Beurkundung ${ }^{43}$ und Kundmachung ${ }^{44}$ auf Anordnung des Bundespräsidenten ${ }^{45}$ durchgeführt wird (Art. 43 und Art. 44 Abs. 3 B-VG). ${ }^{46}$ Objekt ist damit kein Gesetz, sondern ein Gesetzesbeschluss. Wie schon bei der Volksbefragung liegt auch hier die Initiative beim Nationalrat und trägt die Willensäußerung des Bundesvolkes reaktiven Charakter gegenüber einem schon gefassten Beschluss, auf dessen Inhalt das Volk keinen direkten Einfluss nehmen kann. ${ }^{47}$ Insbesondere ist damit kein „Vetoreferendum“48 realisiert worden. Bei fakultativen Referenden über ein einfaches Bundesgesetz muss ein Antrag der einfachen Mehrheit des Nationalrates, ${ }^{49}$ bei solchen über ein Bundesverfassungsgesetz ein solcher eines Drittels der Mitglieder des Nationalrates vorliegen..$^{50}$ Im Fall einer „Gesamtänderung“ der Bundesverfassung, also einer Änderung der Grundprinzipien der Verfassung, ${ }^{51}$ ist

38 Merli, Kommentar zu Art. 41/2 B-VG (Fn. 35), Rz. 10 f.

39 Merli, Kommentar zu Art. 41/2 B-VG (Fn. 35), Rz. 57 ff.

40 Pernthaler, Bundesstaatsrecht (Fn. 4), S. 91.

41 Dazu näher Mayer, Plebiszitäre Elemente (Fn. 14), S. 350 f.

42 Zum Problemkreis näher unten 4 b).

43 Art. 47 B-VG.

44 Art. 49 B-VG.

45 Art. 46 Abs. 1 B-VG.

46 Im Mittelpunkt stehen im vorliegenden Zusammenhang nur die Fälle eines Sachreferendums, nicht eines Personalreferendums. Ein solches sieht die österreichische Bundesverfassung in zwei (in der Praxis bislang irrelevanten) Fällen vor: der Wahl eines Bundespräsidenten im Fall eines einzigen Kandidaten (Art. 60 Abs. 1 B-VG) und im Fall der Abstimmung über seine Absetzung (Art. 60 Abs. 6 B-VG).

47 Merli, Kommentar zu Art. 41/2 B-VG (Fn. 35), Rz. 9.

48 Ein solches könnte von einer bestimmten Anzahl von Personen initiiert werden. Auf landesgesetzlicher Ebene ist ein derartiges Modell in vier österreichischen Bundesländern realisiert: vgl. dazu Pernthaler, Bundesstaatsrecht (Fn. 4), S. 90 m.w.N.

49 Art. 43 B-VG.

50 Art. 44 Abs. 3 B-VG: „Teiländerung“.

51 Dazu schon oben Fn. 4. 
eine Volksabstimmung hingegen obligatorisch durchzuführen. ${ }^{52}$ Die Wirkung der Volksabstimmung ist insoweit am stärksten, als sie rechtlich verbindlich ist - entweder im Sinne einer Annahme des entsprechenden Gesetzesbeschlusses oder der Beendigung des entsprechenden Gesetzgebungsverfahrens. Diese Wirkung erschöpft sich aber nach h.M. ${ }^{53}$ im konkreten Verfahren: Die Volksabstimmung bewirkt weder einen höheren Rang des , abgestimmten“ Gesetzes ${ }^{54}$ noch hindert sie im Fall eines fakultativen Referendums - die Einleitung eines neuen Gesetzgebungsverfahrens mit einem Gesetzesbeschluss gleichen Inhalts. So sehr die Abhaltung eines fakultativen Referendums vor allem bei einfachen Bundesgesetzen eine primär politische Dimension besitzt, da der die Volksabstimmung beschließende Teil in aller Regel ident mit dem das Gesetz an sich beschließenden Teil ist (einfache Mehrheit!), und das Referendum insoweit eine politische Entlastungs- und Systemstabilisierungsfunktion ${ }^{55}$ besitzt, so sehr hat das Institut des obligatorischen Referendums nach Art. 44 Abs. 3 B-VG einen dominierenden verfassungsdogmatischen Gehalt, als es den Angelpunkt des diffusen Begriffes der „Gesamtänderung“ der Bundesverfassung darstellt. ${ }^{56}$ Gemeinsam ist beiden Formen des Referendums wiederum, dass von ihnen jeweils erst einmal Gebrauch gemacht wurde. ${ }^{57}$

\section{e) Die ,Unselbständigkeit“ direkter Demokratie in Österreich}

Allen Formen der direkten Demokratie ist damit gemeinsam, dass sie entweder einem Gesetzgebungsverfahren vorgelagert oder in ein solches als unselbständiger Teil mit notwendigerweise vor- und nachgelagerten Akten eines allgemeinen Vertretungskörpers integriert sind sowie in verfahrensrechtlicher Hinsicht der rechtsstaatlichen Kontrolle durch den VfGH unterliegen. ${ }^{58}$ Insoweit gewinnt die in der österreichischen Dogmatik überwiegend getroffene Aussage, dass keines der direkt-

52 Art. 44 Abs. 3 B-VG.

53 Eingehend $R$. Thienel, Die Rechtswirkungen von Volksabstimmungen nach Art. 43 und 44 B-VG, ÖJZ 1988, 673 ff.; F. Merli, Art. 45, 46 B-VG, in: Korinek/Holoubek, Bundesverfassungsrecht (Fn. 35), Rz. 31 f. m.w.N., a.A. M. Nowak, Politische Grundrechte, 1988, S. 510 ff.

54 V.a. in der Weise, dass eine Änderung dieses Gesetzes ebenfalls einer Volksabstimmung bedürfte.

55 Pernthaler, Bundesstaatsrecht (Fn. 4), S. 86.

56 Dazu näher unten 4 a).

57 Die einzige Volksabstimmung über ein einfaches Bundesgesetz fand am 5.11.1978 im Hinblick auf ein Gesetz betreffend die Inbetriebnahme des einzigen Kernkraftwerkes in Zwentendorf statt. Sie beendete eine jahrelange, überaus emotional geführte Diskussion über die friedliche Nutzung der Kernenergie und ermöglichte der damals im Amt befindlichen SPÖAlleinregierung unter Bruno Kreisky die (wie die weitere politische Entwicklung gezeigt, unschädliche) Erledigung eines (schon aus damaliger Sicht) unpopulären Themas. - Das bislang einzige obligatorische Referendum betraf das Bundesverfassungsgesetz über den Beitritt Österreichs zur Europäischen Union (BGB1. 1994/744) und fand am 12.6.1994 statt. Siehe dazu die Beiträge in A. Pelinka (Hrsg.), EU-Referendum. Zur Praxis direkter Demokratie in Österreich, 1994.

58 Vgl. Art. 141 Abs. 3 B-VG. 
demokratischen Elemente als „Volksgesetzgebung“ im Sinne einer Alternative zur repräsentativ-demokratischen Komponente aufgefasst werden kann (und darf) ${ }^{59}$ und insoweit eine bloß ,semidirekte Demokratie“60 vorliegt, an Konturen.

Es soll in weiterer Folge anhand unterschiedlicher Beispiele illustriert werden, wieso diese bestehenden rechtlichen Strukturen direkter Demokratie an rechtliche und politische Grenzen stoßen. Es lässt sich daraus zeigen, dass - obwohl auf Ebene der Bevölkerung durchaus eine Bereitschaft bestehen würde - auf Seiten der Politik und der Rechtsanwendung eine politische Kultur der direkten Demokratie in Österreich weitgehend fehlt. ${ }^{61}$

\section{Die Rechtsprechung des VfGH und die Systementscheidung gegen direkte} Demokratie

Ein entscheidender Anknüpfungspunkt in dieser Frage ist in Anbetracht marginaler verfassungsrechtlicher Regelungen die dazu ergangene Judikatur. Der VfGH hat zur Bedeutung bzw. Konzeption österreichischer Demokratie in unterschiedlichen Erkenntnissen (Erk.) Stellung genommen. ${ }^{62}$ In diesem Zusammenhang hatte der VfGH auch die Möglichkeit, zur Konzeption der Demokratie im Spannungsfeld zwischen repräsentativer und direkter Demokratie Stellung zu nehmen.

Leading Case in Bezug auf die direkte Demokratie ist dabei ein Erkenntnis über die Regelung der Vorarlberger (vlbg.) Volksbegehren aus dem Jahr 2001 (VfSlg. 16.241/2001). Das Bundesland Vorarlberg hat in seiner Landesverfassung (LVG) die Möglichkeit von Volksbegehren geregelt. Das vlbg. Konzept von Volksbegehren bestand (in seinen wesentlichen Zügen) seit den 1980er Jahren ${ }^{63}$ und wurde vom VfGH aufgrund einer Bekämpfung eines Kostenbescheides im Rahmen eines Volksbegehrens aufgegriffen (Art. 33 Vlbg. LVG). Diese Regelung sah vor, dass „Volksbegehren in Angelegenheiten der Gesetzgebung, die von wenigstens 5000 Stimmberechtigten oder von wenigstens zehn Gemeinden auf Grund von Gemeindevertretungsbeschlüssen gestellt werden, dem Landtag zur Entscheidung darüber vorzulegen (sind), ob er dem Volksbegehren Rechnung tragen will oder nicht." Soweit entsprach die Regelung den Bestimmungen der Bundesverfassung. Darüber hinaus sah die vlbg. Regelung aber vor, dass, wenn der Landtag es ablehnt, ein

59 Zum Volksbegehren vgl. Merli, Kommentar zu Art. 41/2 B-VG (Fn. 35), Rz. 10; zur Volksabstimmung Thienel, Rechtswirkungen (Fn. 53), S. 675 ff.

60 Pernthaler, Bundesstaatsrecht (Fn. 4), S. $83 \mathrm{f}$.

61 I.d.S. etwa auch H. Schäffer, Über die „Schwäche“ der Volksbegehren in Österreich, in: FS Öhlinger, 2004, S. 412 (433): „Was in Österreich letztlich fehlt, ist ein reiferer Umgang mit der direkten Demokratie, nämlich Engagement und Gebrauchnahme von direkt-demokratischen Möglichkeiten auf Seiten der Bürger einerseits, und eine größere Bereitschaft seitens der politischen Parteien, auf erkennbare Anliegen der Bevölkerung einzugehen, insbesondere wenn sie sich schon in Volksbegehren artikuliert haben."

62 Der VfGH urteilt nicht, er „erkennt“; insoweit werden keine Urteile, sondern „Erkenntnisse“ erlassen.

63 Die mit dem Erk. VfSlg. 16.241/2001 aufgehobene Fassung der entsprechenden Bestimmung wurde 1984 geschaffen. 
Volksbegehren, das von wenigstens $20 \%$ der stimmberechtigten BürgerInnen gestellt wurde, umzusetzen, eine Volksabstimmung darüber stattzufinden hat. Wird durch Volksabstimmung das Volksbegehren bestätigt, so ist durch den Landtag (also das Landesparlament), ,dem Volksbegehren Rechnung zu tragen [und ein] dem Volksbegehren inhaltlich entsprechender Gesetzesbeschluß zu fassen“. Durch diese Regelung wurde - entgegen den bestehenden Bestimmungen auf Bundesebene ${ }^{64}$ ein starkes direkt-demokratisches Instrument vorgesehen, das insoweit über das Konzept direkter Demokratie auf Bundesebene hinausging, als es in bestimmten Fällen zu einer Bindung des entsprechenden repräsentativ-demokratischen Organs kommen konnte, worin sich nach Ansicht des VfGH ein Modell einer „Volksgesetzgebung“" (in einem materiellen Sinn) ${ }^{65}$ zeigen würde. Der VfGH erteilte dieser Konzeption eine deutliche Absage und hob die entsprechende Bestimmung als verfassungswidrig auf. Der methodische Ausgangspunkt war dabei, dass die Landesverfassungen nicht der Grundkonzeption der österreichischen Bundesverfassung widersprechen dürfen.

In seinen Kernaussagen ist der VfGH sehr eindeutig: „Das bundesstaatliche Grundprinzip der Bundesverfassung und die damit zwingend verbundene Verfassungsautonomie der Länder (vgl. Art. 99 B-VG) finden ihre Grenze im Kernbereich des repräsentativ-demokratischen Baugesetzes, das nur im Verfahren gemäß Art. 44 Abs. 3 B-VG geändert werden kann (s. VfSlg. 2455/1952). ${ }^{66}$ Eine dem Art. 33 Abs. 6 Vlbg Landesverfassung entsprechende Regelung, der zu Folge der Landtag unter bestimmten Voraussetzungen einen einem Volksbegehren inhaltlich entsprechenden Gesetzesbeschluss zu fassen ,hat', war in der Vlbg. Landesverfassung LGB1. 22/1919 nicht vorgesehen. Die in Prüfung gezogene Bestimmung sieht vor, dass eine von der Mehrheit der Stimmberechtigten unterstützte Gesetzesinitiative auch gegen den Willen (der Mehrheit) des Landtages ,zum Gesetz wird', sie ermöglicht also eine Gesetzeserzeugung sogar gegen den (Mehrheits-)Willen des Parlaments. Eine derartige, Volksgesetzgebung' ist aber mit dem Grundgedanken der repräsentativen Demokratie - mit bloß ausnahmehaft vom Verfassungsgesetzgeber ausdrücklich vorgesehenen direkt-demokratischen Elementen (vgl. VfSlg. 13.500/1993) -, wie er der Bundesverfassung insgesamt zu Grunde liegt, nicht mehr zu vereinbaren. Zu Folge der in Prüfung gezogenen Regelung könnte letztlich jedes beliebige Gesetz im Wege dieser, Volksgesetzgebung erlassen werden. Damit wird aber ein Konkurrenzmodell zum parlamentarischen Gesetzgebungsverfahren konstituiert, das mit dem repräsentativ-demokratischen Grundprinzip der Bundesverfassung nicht mehr vereinbar ist.“

Die Botschaft des VfGH kann damit auf folgende plakative Formel gebracht werden: Die österreichische Bundesverfassung hat - und zwar für alle Ebenen des Bundesstaates - eine Systementscheidung zu Gunsten eines repräsentativen Modells getroffen und direkt-demokratische Elemente als bloße Ausnahme vorgesehen. Di-

64 Vgl. dazu oben $2 \mathrm{c}$ ).

65 Gegen diesen Begriff im vorliegenden Zusammenhang jedoch Pernthaler, Bundesstaatsrecht (Fn. 4), S. 86.

66 Siehe dazu ausführlicher sogleich unter Punkt 4 a). 
rekte Demokratie darf daher auch nicht das repräsentative Modell „aushebeln“, sondern nur untergeordnet ergänzen. Diese Ergänzung ist aber dann überschritten, wenn es zu einer inhaltlichen Bindung eines allgemeinen Vertretungskörpers durch ein in einer Volksabstimmung bestätigtes Volksbegehren komme. Dieser Fall zeigt das Spannungsverhältnis am deutlichsten auf. Der VfGH hat aber bereits davor in unterschiedlichen Zusammenhängen zur Ausgestaltung der österreichischen Demokratie als repräsentative Demokratie mit einer kleinen Nebenrolle direkt-demokratischer Elemente Stellung genommen. ${ }^{67}$ Mit diesem Leading Case erfuhr diese Judikatur für den hier speziellen Zusammenhang eine Präzisierung.

Als zweites Beispiel soll das Erk. zur sog „direkten Abwahl des Bürgermeisters" dienen (VfSlg. 15.302/1998). Dieses Erk. zeigt eine positivere Grundausrichtung gegenüber der direkten Demokratie, auch wenn die konzeptionellen Grenzen, die späterhin zur Aufhebung der Bestimmung im vlbg. Fall geführt haben, bereits deutlich werden. Durch die B-VG-Novelle BGB1. 1994/50468 wurde - wie schon erwähnt ${ }^{69}$ - die Möglichkeit in die österreichische Verfassung aufgenommen, gemäß Art. 117 Abs. 6 B-VG den Bürgermeister in Gemeinden direkt zu wählen. Unterschiedliche Landesverfassungen nahmen in weiterer Folge eine Regelung in Konkretisierung der bundesverfassungsgesetzlichen Bestimmung auf, die auch eine direkte Abwahl des Bürgermeisters zuließ. Der VfGH erkannte, dass durch die bundesverfassungsgesetzliche Einführung des Art. 117 Abs. 6 B-VG, also der Ermöglichung der Direktwahl des Bürgermeisters auf Gemeindeebene, ein Mischmodell aus repräsentativen und direktdemokratischen Elementen entstanden ist, womit auch die direkte Abwahl des Bürgermeisters zulässig ist, selbst wenn dies in der Bundesverfassung nicht explizit vorgesehen ist. Der Anknüpfungspunkt war daher eine spezielle - und hier sogar erst relativ junge - verfassungsgesetzliche Ermächtigung, die es gleichzeitig verhinderte, für die Ebene des Bundes bestehende Restriktionen direkter Demokratie auf die Ebene der Gemeinden zu übertragen.

Nichtsdestotrotz wird die Argumentationslinie des VfGH zur direkten Demokratie und deren Stellung in der österreichischen Verfassung sichtbar: „Der Landesverfassungsgesetzgeber wird durch Art 117 Abs 6 zweiter Satz B-VG ermächtigt, sich - auf Gemeindeebene - für ein Organisationssystem zu entscheiden, das sowohl aus Elementen des parlamentarisch-demokratischen Systems als auch aus Elementen eines Systems direkt-demokratisch legitimierter monokratischer Leitung besteht. Wenn sich der Landesverfassungsgesetzgeber nun für ein solches (duales) System entscheidet - was im Land Salzburg mit der Erlassung des Art 53 Abs 2 LandesVerfassungsG geschehen ist -, dann ist es aber aus der Sicht des Gleichheitssatzes zulässig, auch in jenen Fällen, in denen der Bürgermeister ausnahmsweise vom Gemeinderat gewählt wird, die Abberufung des Bürgermeisters einer vom Gemeinderat initiierten Bürgerabstimmung vorzubehalten. Auf dem Boden der genannten Systementscheidung und im Rahmen des solcherart zugelassenen Systems liegt es nämlich im rechtspolitischen Gestaltungsspielraum des Gemeindeorganisationsgesetz-

67 Siehe etwa VfSlg. 3134/1956, 13.500/1993.

68 In Kraft getreten mit 9.7.1994.

69 Vgl. oben 2 a). 
gebers, für diese Ausnahmsfälle dieselbe Regelung zu treffen wie für den Regelfall des mit direkt-demokratischer Legitimation ausgestatteten Bürgermeisters.“

Für den VfGH ist die direkte Demokratie immer nur eine - letztlich durch Verfassungsbestimmungen im konkreten Kontext ,abgesicherte“ - Ausnahme von der repräsentativen Demokratie. Damit ist eben jenes Systemverständnis der österreichischen Verfassung verbunden, das direkte Demokratie als Ausnahmekonstellation vom Grundsatz vorsieht. Die Entscheidung im Fall der vlbg. „Volksgesetzgebung“ ist dabei allerdings nicht zwingend. Die Kombination unterschiedlicher direkt-demokratischer Elemente unter hohen Auflagen wäre mit der Begründung von direkter Demokratie als gleichwertiges Element ebenso möglich. Im Besonderen ist für die Ausgestaltung direkter Demokratie auf Landesebene die - dem bundesstaatlichen Prinzip als Grundprinzip inhärente ${ }^{70}$ - Verfassungsautonomie der Bundesländer ${ }^{71}$ zu beachten, die den vom VfGH vorgenommenen „Übertragungen“ Restriktionen entgegenbringen könnte. ${ }^{72}$ Der Stellenwert der direkten Demokratie zur Änderung wesentlicher verfassungsrechtlicher Grundsätze ist ein wichtiges Beispiel dafür, dass direkte Demokratie als zentrales Element der Demokratie verstanden werden könnte, auch wenn dies wie bereits dargestellt und anhand von weiteren Beispielen exemplifiziert werden soll, nicht der Fall ist.

\section{Drei Beispiele}

\section{a) Die Gesamtänderung der österreichischen Bundesverfassung und die Volksabstimmung}

Gem Art. 44 Abs. 3 B-VG ist bei einer „Gesamtänderung der Bundesverfassung“ verpflichtend eine Volksabstimmung durchzuführen. Durch diese Bestimmung zeigt sich der Zusammenhang zu Art. 1 B-VG. Die Systementscheidungen der österreichischen Verfassung sind in diesen Fällen nur mit dem österreichischen Bundesvolk zu treffen und nicht durch das Parlament alleine.

Aus der Frage, wann eine Gesamtänderung der österreichischen Bundesverfassung vorliegt, haben sich unterschiedliche Debatten in der österreichischen Rechtswissenschaft entsponnen. Wichtig ist es festzuhalten, dass nach herrschender Lehre der Verfassung Grund- bzw Bauprinzipien innewohnen (Demokratie, Bundesstaat, Republik, Rechtsstaat, Gewaltenteilung und Grundrechte); werden diese Grundprinzipien wesentlich geändert, so liegt eine Gesamtänderung vor. ${ }^{73}$ Auch wenn die Festlegung der Grundprinzipien erst mit den Jahren (vor allem in ihrem Bedeu-

70 Mayer, B-VG-Kommentar (Fn. 3), S. 342 m.w.N.

71 Öhlinger, Verfassungsrecht (Fn. 3), Rz. 233 f. Die Landesverfassungen dürfen nach Art. 99 Abs. 1 B-VG die Bundesverfassung ,nicht berühren“, d.h. ihr nicht widersprechen, innerhalb ihrer Kompetenz haben die Länder aber einen - in diesem Punkt freilich vom VfGH sehr stark restringierten - Gestaltungsspielraum.

72 Zur Kritik an diesem Erk. s. etwa Öhlinger, Grenzen (Fn. 24), S. 402 ff.; Gamper, Demokratie (Fn. 24), S. $441 \mathrm{ff}$.

73 Dazu oben bei Fn. 4. 
tungsgehalt) entstanden ist, ${ }^{74}$ so werden diese als in der Verfassung aus 1920 grundgelegt verstanden. ${ }^{75}$ Die Debatte um die Grundprinzipien der österreichischen Bundesverfassung wurde aber vielmehr aus dem Blickwinkel der Grenzen des sog einfachen Verfassungsgesetzgebers geführt, dem aus Art. 44 Abs. 3 B-VG zumindest prozedurale Grenzen gesetzt werden. ${ }^{76}$ Aus dem Blickwinkel der direkten Demokratie ist von Interesse, dass es bisher nur eine derartige Volksabstimmung anlässlich des EU-Beitritts gegeben hat, ${ }^{77}$ da durch diesen das demokratische, das rechtsstaatliche und das bundesstaatliche Prinzip in ihrem Kerngehalt verändert wurden. Weitere Änderungen des EU- und EG-Vertrages, vor allem auch der Verfassungsvertrag oder der Vertrag von Lissabon, wurden allerdings nicht als Gesamtänderung der Bundesverfassung verstanden. ${ }^{78}$

Die österreichische Verfassung wurde im Laufe der Jahre regelmäßig novelliert; zahlreiche weitere Änderungen der Bundesverfassung wurden in Form sog. Verfassungsbestimmungen und anderer Verfassungsgesetze vorgenommen (in der österreichischen Verfassung besteht kein Inkorporationsgebot). ${ }^{79}$ Alle anderen Änderungen der Verfassung wurden ohne Volksabstimmung durchgeführt, die - wie dargelegt ${ }^{80}$ - auch fakultativ gem Art. 44 Abs. 3 B-VG bei jeder Verfassungsänderung vorgenommen werden könnte. Aus der Nicht-Durchführung von Volksabstimmungen wurde im Übrigen auch formal geschlossen, dass sich die Grundprinzipien der Verfassung durch diese Änderungen nicht geändert haben, ${ }^{81}$ und nicht umgekehrt, dass (vielleicht) eine Notwendigkeit bestanden hätte, die österreichische Bevölkerung in den verfassungsrechtlichen Legislativprozess miteinzubinden. Im Besonderen im Lichte von sukzessive vorgenommenen Verfassungsänderungen, die nicht

74 Dieser Bedeutungsgehalt ist - mangels spezieller verfassungsgesetzlicher Grundlagen - vor allem in der Judikatur des Verfassungsgerichtshofes entwickelt worden. Paradigmatisch kann man dies an der Entfaltung des rechtsstaatlichen Prinzips erkennen: vgl. dazu M. Hiesel, Die Rechtsstaatsjudikatur des Verfassungsgerichtshofes, in: ÖJZ 1999, 522 ff.; ders., Die Entfaltung der Rechtsstaatsjudikatur des Verfassungsgerichtshofs, in: ÖJZ 2009, $111 \mathrm{ff}$.

75 Dazu C. Jabloner, Verfassungsrechtliche Grundordnung und historisch erste Verfassung, in: JRP 2001, 34 ff.; im speziellen Kontext Öhlinger, Verfassungsrecht (Fn. 3), Rz. 75.

76 Die Frage, ob es auch Änderungen des Verfassungsrechts gibt, die nicht einmal durch eine Volksabstimmung vorgenommen werden können (Änderungen des „Verfassungskerns“), wurde in den letzten Jahren heftig diskutiert: vgl. dazu P. Pernthaler, Der Verfassungskern, 1998 und T. Öhlinger, Verfassungskern und verfassungsrechtliche Grundordnung. Gedanken zu Peter Pernthalers Verfassungstheorie, in: FS Pernthaler, 2005, S. 273 ff., einerseits und H. Mayer, Gibt es unabänderliches Verfassungsrecht?, in: FS Schäffer, 2006, S. 473 ff., andererseits.

77 Vgl. dazu Fn. 57.

78 Vgl. die Diskussion zusammenfassend T. Öhlinger, Soll über den Vertrag von Lissabon in Österreich das Volk abstimmen?, in: ecolex 2008, $290 \mathrm{ff}$.

79 Zum Problemkreis und der nach wie vor ausständigen Lösung dieses Problems auf Verfassungsebene K. Lachmayer, Zwischen Ordnung und Chaos - Von der Notwendigkeit der Verfassungsbereinigung und der Illusion des Inkorporationsgebotes, in: JRP 2007, $200 \mathrm{ff}$.

80 Vgl. oben $2 \mathrm{~d}$ ).

81 Zur Frage, wer das Vorliegen einer derartigen Gesamtänderung zu beurteilen hat - der Nationalrat oder der eine Volksabstimmung anordnende (Art. 46 Abs. 1 B-VG) Bundespräsident -, vgl. Merli, Kommentar zu Art. 45, 46 B-VG (Fn. 53), Rz. 10 ff. m.w.N. 
isoliert, aber in ihrer Gesamtheit allenfalls gesamtändernden Charakter besitzen (etwa bedeutenden Kompetenzverschiebungen im Bundesstaat), wurde in diesem Zusammenhang der Begriff einer ,schleichenden Gesamtänderung ${ }^{\text {“82 }}$ geprägt, weil bei all diesen Einzelschritten eine Einbindung der Bevölkerung politisch zumeist nicht gewollt war und ist.

Abschließend stellt sich vor diesem Hintergrund die Frage, ob die Abschaffung der direkt-demokratischen Elemente der Verfassung eine Gesamtänderung wäre. Abgesehen von der zwingenden Volksabstimmung bei der Gesamtänderung selbst könnten alle direkt-demokratischen Elemente aus der Verfassung entfernt und könnte Österreich als bloß repräsentative Demokratie organisiert werden, ohne dass das demokratische Grundprinzip mit seiner Dominanz repräsentativ-demokratischer Komponenten damit in seinem Kerngehalt betroffen wäre. Die Gesamtänderung der Bundesverfassung wäre - wie v.a. das Erk. VfSlg. 16.241/2001 gezeigt hat - viel eher in einer Erweiterung der direkten Demokratie in Österreich gelegen, die sodann einer Volksabstimmung bedürfte. Aber nachdem die diesbezügliche Diskussion bislang fast ausschließlich akademischen Charakter hatte, bleibt diese Frage angesichts der politischen Situation in Österreich ohnedies theoretischer Natur.

\section{b) Zahlreiche Volksbegehren und ihre fehlenden Wirkungen}

Gemäß Art. 41 Abs. 2 B-VG können 100.000 stimmberechtigte BürgerInnen oder je ein Sechstel der stimmberechtigten BürgerInnen dreier Bundesländer einen Antrag an den Nationalrat stellen. Dieser wird als Gesetzesantrag in das Parlament eingebracht (von der Bundeswahlbehörde vorgelegt) und muss von diesem nach den Bestimmungen des Geschäftsordnungsgesetzes ${ }^{83}$ behandelt werden. Daraus ergibt sich allerdings keine inhaltliche Vorgabe für den Nationalrat. Dieser kann vielmehr das Thema in weiterer Folge gar nicht regeln oder auch ablehnen. ${ }^{84}$ Das Volksbegehren schafft daher nur prozedurale, aber keine materiellen Vorgaben für das Parlament.

In historischer Perspektive haben sich bei diesem Instrument die rechtlichen Rahmenbedingungen verbessert. Die Verfassung aus 1920 sah eine noch restriktivere Regelung mit 200.000 Stimmberechtigten bzw. der Hälfte der Stimmberechtigten

82 Dazu etwa Öhlinger, Verfassungsrecht (Fn. 3), Rz. 72.

83 Vgl. dazu näherhin Öhlinger, Verfassungsrecht (Fn. 3), Rz. 452. Vgl. insb. § 24 Geschäftsordnungsgesetz 1975 BGBl. 1975/410: „(1) Bei Festlegung der Tagesordnung des Nationalrates haben Volksbegehren den Vorrang vor allen übrigen Gegenständen.

(2) Die Vorberatung eines Volksbegehrens hat innerhalb eines Monates nach Zuweisung an den Ausschuß zu beginnen. Nach weiteren vier Monaten ist dem Nationalrat jedenfalls ein Bericht zu erstatten.“

84 Es werden zwar durchaus unterschiedliche Meinungen vertreten, die herrschende Meinung sieht eine - gerichtlich freilich nicht durchsetzbare - Verpflichtung vor, dass der Nationalrat das Volksbegehren formal behandeln muss, aber keinen förmlichen Ablehnungsbeschluss in jenen Fällen zu fassen hat, in denen er dem Volksbegehren nicht zu entsprechen gedenkt. Dazu m.w.N. Merli, Kommentar zu Art. 41/2 B-VG (Fn. 35), Rz. 57. 
dreier Länder vor. Überdies musste das Volksbegehren zwingend in Form eines Gesetzesantrages eingebracht werden. ${ }^{85}$ Diese Bedingungen wurden in mehreren Schritten geändert: zum einen durch die mit der B-VG-Novelle BGBl. 1981/350 vorgenommene Herabsetzung auf die notwendige Unterstützung durch nunmehr 100.000 Stimmberechtigte bzw. von je einem Sechstel dreier Länder ${ }^{86}$, zum anderen durch die mit der B-VG-Novelle BGBl. 1988/685 realisierte Ermöglichung eines Volksbegehrens, das nicht schon einen ausformulierten Gesetzesentwurf, sondern allenfalls auch eine bloße Anregung beinhalten kann. ${ }^{87}$ Die meisten Volksbegehren, deren praktische Entwicklung erst 1964, also knapp 20 (!) Jahre nach Beginn der Zweiten Republik begann, dienten der Artikulation von Anliegen für oder gegen größere Infrastrukturprojekte wie etwa die Errichtung von Kraftwerken oder die Anschaffung und Stationierung von Abfangjägern, der Mobilisierung von sozialen Anliegen (etwa Pensionsregelungen, „Politikerprivilegien“, Sozialstaat) oder sonst breitenwirksamen (zum Teil auch massenmedial sehr stark forcierten) Initiativen (Unabhängigkeit des Rundfunks, ${ }^{88}$ Tierschutz, Gentechnik, atomare Sicherheit, Neutralität, Anti-EU-Volksbegehren, Erhaltung von Postämtern). ${ }^{89}$

Die rechtlich schwache Ausgestaltung von Volksbegehren in Österreich wirkt sich nicht zuletzt auch in der politischen Realität aus. In Österreich gab es seit den 1960er Jahren 33 Volksbegehren. ${ }^{90}$ Nur knapp ein Drittel dieser Volksbegehren wurde im Nationalrat dergestalt ,,angenommen“, dass es zu unmittelbaren gesetzlichen Änderungen kam. Der größte Teil wurde entweder vom Parlament (in Gestalt eines entsprechenden Ausschussberichts) abgelehnt oder nicht behandelt.

Was ist dann die demokratiepolitische Funktion dieser Volksbegehren? Sie schaffen öffentliche Diskussionen zu Themen bzw. dienen auch - v.a. Oppositionsparteien - zu dem Zweck, für oder gegen bestimmte Themen Stimmung zu machen,

85 Art. 41 Abs. 2 B-VG i.d.F. BGBl. 1920/1: ,Jeder von 200.000 Stimmberechtigten oder von je der Hälfte der Stimmberechtigten dreier Länder gestellte Antrag (Volksbegehren) ist von der Bundesregierung dem Nationalrat zur geschäftsordnungsmäßigen Behandlung vorzulegen. Das Volksbegehren muß in Form eines Gesetzentwurfes gestellt werden.“

86 Letztere Variante blieb stets eher theoretischer Natur, weil je ein Sechstel der Stimmberechtigten schon der drei kleinsten österreichischen Bundesländer weit über 100.000 Personen darstellen würden.

87 Seither muss das Volksbegehren - aus Kompetenzgründen nahe liegend - eine durch Bundesgesetz zu regelnde Angelegenheit betreffen, kann jedoch in Form eines Gesetzesantrages gestellt werden.

88 Das Volksbegehren „Rundfunk-Reform“ im Jahr 1964 war nicht nur das erste (und mit 832.353 Eintragungen eines der erfolgreichsten) Volksbegehren in der Zweiten Republik, sondern im Unterschied zu anderen insoweit erfolgreich, als es - wenn auch mittelbar - zur Erlassung eines Rundfunkgesetzes führte, das dem Österreichischen Rundfunk (ORF) formal von den politischen Parteien weitgehend unabhängige Strukturen verschaffte (,Rundfunkreform 1967“).

89 Vgl. H. Widder, Die plebiszitäre Komponente im Gesetzgebungsverfahren, in Österr. Parlamentarische Gesellschaft (Hrsg.), 75 Jahre Bundesverfassung, 1995, S. 315 (318ff.); siehe dazu auch Merli, Kommentar zu Art. 41/2 B-VG (Fn. 35), Rz. 14.

90 Eine Gesamtdarstellung aller Volksbegehren in der Zweiten Republik findet sich im Internet unter http://www.bmi.gv.at/cms/BMI_wahlen/volksbegehren/Alle_Volksbegehren.aspx (31.8.2009). Vgl. dazu auch die Übersicht bei Schäffer, Schwäche (Fn. $\overline{6} 1$ ), S. 415 ff. 
also die Bevölkerung zu mobilisieren. ${ }^{91}$ Dies kann auch im Kontext von Wahlen einen entsprechenden Beitrag zur öffentlichen Diskussion bilden und für sich genommen eine Bereicherung des demokratischen Diskurses darstellen. Eine darüber hinausgehende Bedeutung, vor allem die durch die Verfassung vorgesehene Idee, Gesetze zu initiieren, konnte das Instrument der Volksbegehren bisher nicht erfüllen. Es bleibt damit realiter einen Schritt vor der verfassungssystematischen Positionierung als Gesetzesinitiative neben Bundesregierung und dem National- und Bundesrat selbst (vgl. Art. 41 Abs. 1 B-VG ${ }^{92}$ ) stehen.

Eine Weiterentwicklung des Instruments des Volksbegehrens kann sich vor diesem Hintergrund nur in kleinen Schritten vollziehen. Ein solcher ist etwa mit der B-VG-Novelle BGBl. I 2009/31 vorgenommen worden. Durch diese wurde Art. 28 Abs. 4 B-VG angepasst und dabei folgende Passage aufgenommen: „Mit dem Beginn einer neuen Gesetzgebungsperiode gelten vom Nationalrat der vorangegangenen Gesetzgebungsperiode nicht erledigte Volksbegehren und an den Nationalrat gerichtete Bürgerinitiativen als Verhandlungsgegenstände des neu gewählten Nationalrates." Damit werden Volksbegehren nicht mehr durch den Ablauf der Legislaturperiode - angesichts des Grundsatzes der „Diskontinuität“ zwischen den Legislaturperioden des Nationalrates ${ }^{93}$ - automatisch hinfällig. Es handelt sich damit um eine bescheidene verfassungsgesetzliche Weichenstellung für die verfahrensrechtliche Aufwertung von Volksbegehren. Aber auch bei Betrachtung der Materialien ${ }^{94}$ und der parlamentarischen Debatte ${ }^{95}$ zu dieser Änderung zeigt sich, dass diese Bestimmung als technische Verbesserung verstanden, aber nicht in den unmittelbaren Kontext der direkten Demokratie gestellt wurde. Die Rolle der direkten Demokratie in Österreich ändert sich durch die neue Bestimmung nicht, sehr wohl trägt sie aber zu einer Effektuierung dieses Instruments bei, weil es damit nicht mehr zu einem - rechtspolitisch mit Recht kritisierten ${ }^{96}$ - „Versickern“ eines Volksbegehrens im Rahmen der parlamentarischen Behandlung ${ }^{97}$ im Vorfeld von Neuwahlen zum Nationalrat kommen kann.

91 Dies wurde v.a. im Fall des Volksbegehrens „Österreich zuerst“ („Ausländervolksbegehren“) im Jahr 1993 deutlich, das von der rechtspopulistischen FPÖ unter Jörg Haider initiiert wurde.

92 „Gesetzesvorschläge gelangen an den Nationalrat als Anträge seiner Mitglieder, des Bundesrates oder eines Drittels der Mitglieder des Bundesrates sowie als Vorlagen der Bundesregierung."

93 E contrario Art. 28 Abs. 1 S. 1 B-VG.

94 IA 487/A AB 94 BlgNR 24. GP.

95 Nationalrat, XXIV. GP, 11.3.2009, 16. Sitzung des Nationalrates (im Stenographischen Protokoll), 232-235.

96 Dazu Mayer, Plebiszitäre Elemente (Fn. 14), S. 350 f.; Merli, Kommentar zu Art. 41/2 B-VG (Fn. 35), Rz. 61.

97 Zur alten Rechtslage siehe noch Schäffer, Schwäche (Fn. 61), S. 431. 


\section{c) Diskussion um Geschworenengerichte im Strafprozess}

Auch in der Gerichtsbarkeit findet sich durch Art. 91 B-VG eine verfassungsgesetzliche Grundlage für eine Institution der direkten Demokratie in Gestalt der Mitwirkung des Volkes an der Staatsfunktion Rechtsprechung. Dabei hält wiederum proklamatorisch Art. 91 Abs. 1 B-VG fest, dass ,[d] as Volk [...] an der Rechtsprechung mitzuwirken" hat. Konkretisiert wird dies durch Abs. 2 leg. cit. in Bezug auf die Geschworenengerichtsbarkeit („Bei den mit schweren Strafen bedrohten Verbrechen, die das Gesetz zu bezeichnen hat, sowie bei allen politischen Verbrechen und Vergehen entscheiden Geschworene über die Schuld des Angeklagten.") und Abs. 3 leg. cit. in Bezug auf die Schöffengerichtsbarkeit. Die BürgerInnen sind dabei nicht Vertreter eigener Interessen, sondern Vertreter des Volkes, die zusammen mit den Richtern Funktionen der Gerichtsbarkeit ausüben. ${ }^{98}$

In der aktuellen politischen Debatte geht es vor allem um die Reform der Geschworenengerichtsbarkeit. Dabei wird diese als Belastung verstanden, und es werden die Grenzen der Leistungsfähigkeit des Modells angesichts der Beteiligung von Laien in zum Teil relativ komplexen Verfahren aufgezeigt. ${ }^{99}$ Durch einen wenig diskutierten, dafür umso schneller realisierten gesetzgeberischen Schritt der jüngsten Zeit wurde nunmehr der Anwendungsbereich der Geschworenengerichtsbarkeit auf einfachgesetzlicher Ebene bedeutend reduziert. ${ }^{100}$ Daneben stehen auch verfahrensrechtliche Änderungen der Geschworenengerichtsbarkeit - durchwegs im Sinne einer verstärkten Rolle des jeweiligen Berufsrichters - weiter zur Diskussion. Die direkte Demokratie in ihrer Gestalt der Mitwirkung an der Gerichtsbarkeit steht damit zum gegenwärtigen Zeitpunkt auf mehr als tönernen Füßen.

\section{Politisch-kultureller Kontext der „schwachen“ direkten Demokratie}

Beleuchtet man die Rolle direkt-demokratischer Instrumente in der österreichischen Verfassung, so kommt man nicht umhin, den sozio-politischen Kontext dieser Regelungen, m.a.W.: die Verfassungsrealität, in den Blick zu nehmen. Diese zeigt ein starkes repräsentativ-demokratisches System, das jahrzehntelang von der Dominanz der beiden großen Parteien SPÖ und ÖVP im Rahmen einer „Großen Koalition“, die mit wenigen zeitlichen Ausnahmen stets über die für Verfassungsänderungen erfor-

98 Pernthaler, Bundesstaatsrecht (Fn. 4), S. 94.

99 Dazu kritisch M. Burgstaller, Argumente für die Geschworenengerichtsbarkeit, in: JB1. 2006, $69 \mathrm{ff}$.

100 Vgl. dazu die durch Art. 18 Z. 4 lit. b des Budgetbegleitgesetzes 2009 BGB1. I 2009/52 bewirkte Änderung von $\S 31$ Abs. 2 Z. 1 Strafprozessordnung (StPO). Seither unterliegen dem jeweiligen Landesgericht als Geschworenengericht nur mehr Straftaten, die mit lebenslanger oder einer Freiheitsstrafe bedroht sind, deren Untergrenze mehr als (und nicht mehr - wie zuvor - mindestens) fünf Jahre und deren Obergrenze mehr als zehn Jahre beträgt. Dies führt zu einer bedeutenden Einschränkung des Anwendungsbereichs der Geschworenengerichtsbarkeit. 
derliche Zwei-Drittel-Mehrheit verfügte, ${ }^{101}$ geprägt war und in dem diese parlamentarische Dominanz der Parteien durch die „Sozialpartnerschaft" ${ }^{\text {"102 }}$ mit ihren Vorfeldorganisationen auch den vor- und nebenparlamentarischen Raum erfasste. Initiativen zur Gesetzgebung kamen vor allem im Zusammenwirken dieser Sozialpartner zustande und wurden daher überwiegend parlamentarisch bloß formal nachvollzogen, ja es wird sogar ein Element einer ,gesellschaftlichen Selbstverwaltung "103 genau in dieser Sozialpartnerschaft gesehen und insoweit die Partizipation auf der Ebene der Vollziehung durch diesen Bereich erfasst.

Diese weite Bereiche der Zweiten Republik prägende, in neuerer Zeit aber oftmals kritisch hinterfragte Sozialpartnerschaft als korporatistisches Element, ${ }^{104}$ das sich als institutionelles Substitut für die Zivilgesellschaft darstellt, machte - aus der Perspektive der politischen Machtträger - direkt-demokratische Initiativen im weitesten Sinne entbehrlich, weil die einzelnen Sozialpartner ohnedies alle Bevölkerungsgruppen erfassten. Bei einem solchen Selbstverständnis blieb aber kein von den Parteien akzeptierter Platz für direkte Demokratie, womit - wie dargestellt - die direkte Demokratie im Abseits blieb.

\section{Conclusio - Stand und Perspektiven direkter Demokratie in Österreich}

Betrachtet man die Situation in Österreich gesamthaft, so zeigt sich, dass direkte Demokratie in Österreich weder in rechtlicher noch in politischer Hinsicht einen großen Stellenwert hat. Sie erweist sich insoweit als für den rechtlichen und politischen Prozess nicht relevant. Obwohl unterschiedliche rechtliche Instrumente bestehen, um direkt-demokratische Aktivitäten zu setzen, besteht sowohl auf rechtlicher als auch auf politischer Ebene auch ein gewisses Misstrauen gegen direkt-demokratische Elemente.

Dies hat auch seine historischen Wurzeln. Vor allem die Absage an die Demokratie durch den faschistischen Ständestaat $1934^{105}$ und die (manipulierte) Volksabstimmung aus 1938, die zum „Anschluss“ geführt hat, nährten diese Skepsis gegenüber direkt-demokratischen Elementen. Geschworenengerichte nach dem Zweiten Weltkrieg haben dazu geführt, dass Verurteilungen unterblieben sind, und Anti-

101 Vgl. Art. 44 Abs. 1 B-VG.

102 Zu dieser etwa Öhlinger, Verfassungsrecht (Fn. 3), Rz. 369 f.

103 Siehe dazu etwa $P$. Oberndorfer, Die Tätigkeit der Wirtschaftskammern als gesellschaftliche Selbstverwaltung, in: FS 60 Jahre Wirtschaftskammer Österreich, 2006, S. 87 (94 ff.).

104 Zu den Reformtendenzen der Sozialpartnerschaft aus politikwissenschaftlicher Sicht F. Karlhofer, Die Wirtschaftskammer als korporativer Akteur, in: FS 60 Jahre Wirtschaftskammer Österreich, 2006, S. 65 ff.; in rechtsvergleichender Sicht vgl. M. Fink, Staat-Verbände-Beziehungen im 21. Jahrhundert: Das neokorporatistische Modell am Ende?, ebda., S. $19 \mathrm{ff}$.

105 Dazu jüngst L. Dvorak, „Das Parlament gehört nicht hierher“ - Die Vorbereitungen zum Staatsstreich gegen die Verfassung 1932 - 1934 in ausgewählten Quellen, in: juridikum 2009, $41 \mathrm{ff}$. 
EU-Volksbegehren zeigen in aller Regel schon im Ansatz keine konstruktiven Lösungen auf. ${ }^{106}$

Die mit der direkten Demokratie verbundenen negativen Erfahrungen reflektieren Ängste, die aus mangelnder Verfassungskultur im Umgang mit direkter Demokratie entstanden sind. Dabei sind die Missbrauchsmöglichkeiten und Gefahren im Rahmen des repräsentativ-demokratischen Systems im gleichen Ausmaß gegeben. Die in Österreich entstandene politische Ignoranz gegenüber direkter Demokratie spiegelt sich in ihrer rechtlichen Irrelevanz wider. Um diesen Zustand zu ändern, bedürfte es daher zu aller erst eines politischen Verständnisses der direkten Demokratie und eines positiven Diskurses über ihre Möglichkeiten und Chancen. Nur in einem solchen Umfeld könnten die bereits bestehenden verfassungsrechtlichen Optionen direkter Demokratie ihre Funktion erfüllen. Die Bereitschaft zu einem diesbezüglichen Umdenken ist allerdings nicht in Sicht ...

106 Siehe zur Problematik der Verwendung der direkten Demokratie für populistische Zwecke M. Stelzer, Direkt-demokratische Elemente in der österreichischen Verfassung - eine rechtsvergleichende Betrachtung, in: FS Hartmut Maurer, 2001, S. 1119 (1130 f.). 